\title{
Simultaneous assessment of Lagrangian strain and temperature fields by improved IR-DIC strategy
}

\author{
X. G. Wang, C. H. Liu, C. Jiang*
}

\begin{abstract}
:
This paper focuses on the development of a novel digital image correlation strategy based on infrared imaging (IR-DIC) for realizing simultaneous assessment of Lagrangian strain and temperature fields. A major difficulty in the IR-DIC is the evolution of the thermal field during deformation that is unexpected in the image correlation. Two solutions are proposed to tackle this difficulty. The first solution is to utilize a high-pass filter to eliminate the variation part of the thermal signal, and the second solution is to employ an advanced metric, the mutual information, as the correlation criterion. Both methods are verified through a tensile test performed on a notched specimen. The estimated displacement and strain fields demonstrate well the desirable performance of the proposed methods. Thanks to the kinematic field assessment, the obtained thermal fields can be described in the Lagrangian coordinate system, thus the temperature evolution of the material points during deformation can be followed effectively.
\end{abstract}

Keywords: Digital image correlation; Infrared thermography; Lagrangian temperature; Strain measurement; Mutual information 


\section{Introduction}

In recent decades, some powerful and flexible non-interferometric optical techniques have been developed in experimental mechanics [1]. A representative technique is the digital image correlation (DIC) method [2-4] that enables full-field mapping of displacement and strain in deforming materials. Another useful optical method is the infrared thermography (IRT) [5-7] allowing full-field temperature measurement. Both techniques possess the advantages of noncontact, real-time and full-view. They permit the experimental assessment of strain and temperature fields that, in a conventional sense, can only be numerically simulated using, for instance, finite element method (FEM). It should be emphasized that these optical methods do not have stringent requirements on the experimental conditions as the traditional interferometric approaches, and they can be applied to a broad range of spatial resolution from macroscale to microscale [8-10]. Thanks to these advantageous features, the DIC and IRT methods have been successfully used in the study of material deformation behaviors. It shows that they are particularly useful in investigating the inhomogeneous deformation and strain localization phenomena, e.g., Lüders and Portevin-Le Châtlelier banding [11-15], crystal slip [16-18], fatigue damage [19-22] and crack propagation [23, 24]. In most cases, the DIC and IRT techniques are used independently, thus the kinematic and thermal features of the material deformation response are characterized separately. The recent investigations [25-27] show that the combination of the two techniques can provide enriched information and further insight on the material behaviors based on a complete characterization of both kinematic and thermal aspects.

Presently, there exist several technical solutions for realizing the integration of DIC and IRT for a kinematic-thermal coupled measurement. The first consideration was through the simple combination of the two imaging systems in which each camera (visible-light/IR) observes one surface of the specimen, i.e., the two-face setup [25, 27-31]. In this case, the specimen is preferred to be sufficiently thin such that the strain and thermal gradients through the specimen thickness can be neglected. An alternative configuration is the so-called oneface setup [32-36] that employs the two cameras to observe the same surface of the specimen. The challenge of this setup lies that a special speckle coating is normally required which should be considered both suitable in the strain assessment by DIC and temperature 
measurement by IRT [32]. Besides, a common technical difficulty for the above two-camera setups is that synchronization is needed for the two image acquisition systems for realizing the temporal correspondence of data. And the spatial alignment of the recorded visible-light and IR images (due to the differences in camera resolution, location and field of view) should be also ensured through some dedicated calibration methods [26, 27, 36]. Recently, some researchers [37-39] proposed to perform the strain and temperature field measurements simultaneously using a single IR camera. In this way, the DIC computation is based on the IR images but not the traditional visible-light images, so-called IR-DIC. This solution allows circumventing the difficulty of the synchronization and spatial calibration in the two-camera configuration. It is, therefore, preferred by the investigators due to its simplicity.

Nevertheless, several issues should be addressed before an appropriate utilization of the IRDIC method. First, the DIC calculation is based on the images with speckled pattern that is assumed to be consistent during the deformation process. However, the speckled pattern is reflected by thermal signal (e.g., radiative flux) in IR images which becomes variable during the deformation due to thermoelasticity and/or thermoplasticity (i.e., intrinsic dissipation). This effect should be taken into account in the image processing of the DIC computation. Second, the accuracy of the temperature measurement depends on the exact knowledge of the specimen surface emissivity. The raw measured thermal fields are depicted in Euler coordinate system such that the emissivity of the moving material points cannot be followed during deformation. It is the drawback of the traditional Euler thermography. Nevertheless, the kinematic fields estimated by the IR-DIC enable the thermal field to be transformed from Euler to Lagrangian coordinate system, so-called the Lagrangian thermography [26]. Thus, potentially the temperature field evolution of the material points can be evaluated properly based on the initial emissivity field in the undeformed configuration. The authors consider that the two issues above-mentioned are crucial to the precision of both the strain and temperature estimation by IR-DIC. It is the focus of the present investigation.

This paper is organized as follows. First, a general principle of the IR-DIC method is introduced. And the improved strategy of IR image processing is proposed, in which two feasible solutions are elucidated. Second, the material and experimental set-up are described. Then the Lagrangian strain and temperature field assessments are realized by using the 
developed methods. The obtained results are presented and discussed. Finally, some summary remarks are given in the conclusion.

\section{Methodology}

\subsection{Principle of IR-DIC}

DIC is a method of image registration operating on the images with speckled pattern. In the practical application of strain measurement, it necessitates a speckled coating applying to the specimen surface that will deform together with the specimen when subjected to the mechanical load. The recorded images of specimen surface function, therefore, as the carrier of deformation information, from which the displacement and strain fields can be estimated by the DIC algorithm. The classical DIC method is applicable to the visible-light images with speckles in good white-black contrast. The gray level of the pixels is considered as the unique signature of the material points that is supposed to be constant during the deformation according to the "conservation of optical flow" [40]. In an analogous way, the principle of DIC is also applicable to the IR images though the thermal signal is sampled here instead of the gray level. The raw thermal signal of a modern IR camera is the digital level (DL) that can be converted into radiative flux through the calibration by a commercial black body. A distinct feature of the radiative flux is that it depends on two factors: apparent emissivity and surface temperature. Generally, the emissivity is considered as an intrinsic characteristic of the material, and it is assumed to be constant during the deformation as the same as the gray level. The temperature may also have an influence on the radiative flux. It is, however, considered as a secondary factor here that will be discussed later. In principle, the only difference between the classical DIC and IR-DIC is the difference in wavelength of the acquired radiation (visible light and infrared) but without genuine difference relative to the image correlation. Hence, the hypothesis of the conservation of optical flow is considered reasonable to the IR-DIC based on radiative flux measurement.

In the present study, the powerful image registration toolbox "elastix" [41] is adopted for the DIC computation [18]. Two images are involved in DIC that characterize the undeformed and deformed state of the specimen surface subjected to a known load. An IR image is a scalar function of the spatial coordinate $x$ that gives the radiative flux at each discrete pixel, 
denoted by $\Phi(x)$. The image of the undeformed state is called as the reference image $\Phi_{r e f}(x)$, and that of the deformed state the deformed image $\Phi_{\text {def }}(x)$. A displacement field $u(x)$ is necessary to be introduced that allows correlating the two images under the conservation of optical flow, thus one can obtain

$$
\Phi_{r e f}(x)=\Phi_{d e f}[x+u(x)]=\Phi_{d e f}[R(x)]
$$

where $R(x)=x+u(x)$ is defined as the transformation function, and $\Phi_{d e f}[R(x)]$ is the transformed deformed image (called as "transformed image" for short in the text).

The objective of the image correlation is to determine a transformation $R(x)$ that makes $\Phi_{d e f}[x+u(x)]$ spatially aligned to $\Phi_{r e f}(x)$. The quality of the alignment is evaluated by a similarity measure $S$. For the case of nonrigid transformation, this problem is often ill-posed, thus a penalty term $P$ is necessary to be introduced to constrain the transformation $R$. Generally, the image correlation problem is constructed as an optimization problem with the introduction a cost function $C$. The goal is to minimize the $\operatorname{cost} C$ with respect to $R$

$$
\begin{gathered}
\text { ? } \arg \min C\left[R ; \Phi_{r e f}(x), \Phi_{d e f}(x)\right] \\
C\left[R ; \Phi_{r e f}(x), \Phi_{d e f}(x)\right]=-S\left[R ; \Phi_{r e f}(x), \Phi_{d e f}(x)\right]+\gamma P(T)
\end{gathered}
$$

where $\gamma$ is a factor weighting similarity against regularity.

The measure of the similarity $S$ is actualized by predefining some kind of correlation criterion or commonly called the metric in image registration. Among the different correlation criteria, the normalized cross-correlation (NCC) is the one mostly adopted in the material deformation characterization $[3,18]$. This criterion assumes a linear relation between the intensity values of the reference image and deformed image. It can be defined relative to the thermal signal by

$$
S_{N C C}=\frac{\sum_{x_{i} \in \Omega_{Z}}\left(\Phi_{r e f}\left(x_{i}\right)-\bar{\Phi}_{r e f}\right)\left(\Phi_{d e f}\left[R\left(x_{i}\right)\right]-\bar{\Phi}_{d e f}\right)}{\sqrt{\sum_{x_{i} \in \Omega_{Z}}\left(\Phi_{r e f}\left(x_{i}\right)-\bar{\Phi}_{r e f}\right)^{2} \sum_{x_{i} \in \Omega_{Z}}\left(\Phi_{d e f}\left[R\left(x_{i}\right)\right]-\bar{\Phi}_{d e f}\right)^{2}}}
$$


with $\Omega_{Z}$ the domain of the reference image $\Phi_{r e f}(x)$, and the average radiative flux values $\bar{\Phi}_{r e f}=\frac{1}{\left|\Omega_{z}\right|} \sum_{x_{i} \in \Omega_{z}} \Phi_{r e f}\left(x_{i}\right) \quad \bar{\Phi}_{\text {def }}=\frac{1}{\left|\Omega_{z}\right|} \sum_{x_{i} \in \Omega_{z}} \Phi_{d e f}\left[R\left(x_{i}\right)\right]$ where $\left|\Omega_{z}\right|$ denotes the number of pixels.

The cost function $C$ is equivalent to the minus $S$ in the case that the penalty term $P$ is absent. Commonly, the parametric approach is adopted to minimize the cost function. In this way, the number of possible transformations is constrained by introducing a parameterization model of the transformation. Then the original optimization problem in Eq. (2) becomes

$$
R_{\pi}=\arg \min C\left[R_{\mu} ; \Phi_{r e f}(x), \Phi_{d e f}(x)\right]
$$

where the subscript $\mu$ indicates that the transformation $R$ is parameterized.

The parameterized transformation $R_{\mu}$ defines what type of deformation is expected between the reference image and deformed image. As the strain heterogeneity is inevitable in the deformation of materials and structures, a transformation model capable of describing a freeform deformation is generally desired. The extensive studies $[18,41-43]$ demonstrate that the B-spline function is appropriate in characterizing a heterogeneous deformation process. In elastix the B-spline function is parameterized by

$$
R_{\mu}(\boldsymbol{x})=\boldsymbol{x}+\sum_{x_{k} \in N_{x}} p_{k} \beta^{3}\left(\frac{\boldsymbol{x}-\boldsymbol{x}_{k}}{q}\right)
$$

where $x_{k}$ represents the control points, $\beta^{3}(x)$ the cubic multidimensional B-spline polynomial, $p_{k}$ the B-spline coefficient vectors, $e$ the B-spline control point spacing, and $N_{x}$ the set of all control points in the compact support of the B-spline at the location $x$.

In the conventional DIC implementation based on visible-light images, the B-spline transformation is normally competent in modelling a heterogeneous deformation under the common NCC criterion. Here an underlying prerequisite is that the gray level of specimen surface only shows a gradual and linear evolution during the deformation process without abrupt changes. This requirement may, however, do not hold true in the case of the IR-DIC based on radiative flux measurement. As formerly mentioned, the radiative flux of specimen surface depends not only on the emissivity distribution but also on the temperature field. The former is relatively stable during the deformation as an intrinsic characteristic of the material 
points, but the latter is definitely a variable due to thermoelastic or thermoplastic effect. The direct adoption of the NCC criterion without considering the above effect will lead to a deviation from the basic hypothesis of the conservation of optical flow, and then may produce nonnegligible errors in the displacement and strain estimation. To handle this problem, two possible solutions are proposed in the present study. The first solution is to resort to some filtering technique that is expected to be able to eliminate the influence of temperature variation on the radiative flux. The second option is focused on the correlation criterion. It necessitates a more advanced and versatile metric capable of treating the image correlation even under the unfavorable condition that the conservation of optical flow is relative. The specification of the two proposed solutions are presented in the following sections.

\subsection{The first solution: image filtering}

The IR camera is, in essence, a "photon counting device". Depending on the amount of photons acquired, it returns the electrical voltage before being converted into numerical signal, i.e., the DL. The photon-volt-DL transfer functions are linear functions. Finally the DL needs to be converted into radiative flux or temperature through calibration. The StefanBoltzmann law describes the radiative flux $\Phi$ emitted from a black body in terms of its temperature $T$

$$
\Phi=\sigma T^{4}
$$

where $\sigma\left(=5.67 \times 10^{-8} \mathrm{~W} \mathrm{~m}^{-2} \mathrm{~K}^{-4}\right)$ represents the Stefan-Boltzmann constant, and $T$ is the absolute temperature of the black body in degree Kelvin (K) with $\mathrm{K}={ }^{\circ} \mathrm{C}+273.16$.

A body that does not absorb all incident radiation emits less total radiation than a black body and is characterized by an emissivity $\varepsilon$. Then its radiative flux can be expressed by

$$
\Phi=\varepsilon \sigma T^{4}
$$

Practically, most of the engineering materials are considered as the gray body which is idealized to possess constant emissivity over all wavelengths and temperatures. Here, concerning the current issue of IR image correlation, the emissivity is assumed to be invariant during the material deformation for a given physical point. Then the problem becomes how to eliminate influence of temperature variation on the total $\Phi$ so as to meet the basic assumption 
of the "conservation of optical flow". In this case, the radiative flux for a given moment $t$ during the material deformation can be decomposed into two parts

$$
\Phi(t)=\Phi_{i n i}+\Phi_{v a r}(T, t)
$$

In the above equation, $\Phi_{i n i}$ represents the radiative flux at the initial undeformed state, i.e., that of the reference thermal image, then $\Phi_{i n i}=\Phi_{r e f}$. And $\Phi_{v a r}$ denotes the variation part of the radiative flux that is a function of temperature and time. Here we suppose that the part of $\Phi_{v a r}$ is possessed of some special characteristics in the frequency domain that is possible to be disassociated from the total $\Phi$ with the assistance of some spectral decomposition technique. The rationality of this hypothesis is based on the fact that the temperature always tends to be uniform due to thermal conduction, especially for metals. Thus it will lead to a low frequency signal $\Phi_{v a r}$, which could be in contrast with the high frequency signal $\Phi_{i n i}$ in light of the heterogeneous nature of the speckled coating on the specimen surface (i.e., a heterogeneous emissivity map). Now this problem becomes how to design a high-pass filter to filter out the low frequency $\Phi_{v a r}$ in the measured thermal fields. To this end, a Butterworth filter [44] is designed to realize the high-pass filtering. A predominant feature of the adopted filter is that it enables a very flat frequency response without ripples in the passband.

The implementation of the high-pass filtering relative to a two-dimensional deformed thermal image $\Phi_{d e f}(x, y)$ can be summarized as the following steps.

(1) The thermal image $\Phi_{d e f}(x, y)$ is first transformed from the spatial domain into the frequency domain by using the discrete Fourier transform. A function $F(w, z)$ is introduced to represent the transformed image in the frequency domain, then one can obtain

$$
F(w, z)=\frac{1}{M N} \sum_{x=0}^{M-1} \sum_{y=0}^{N-1} \Phi_{d e f}(x, y) e^{-j 2 \pi(w x / M+z y / N)}
$$

where $M$ and $N$ denote the total numbers of pixels along the horizontal and vertical directions of the thermal image, respectively.

(2) The obtained $F(w, z)$ is then multiplied by the Butterworth filter function $H(w, z)$, it results a filtered image $G(w, z)$ in the frequency domain

$$
G(w, z)=H(w, z) F(w, z)
$$

with the definition of the filter function 


$$
H(w, z)=\frac{1}{1+\left[\frac{D_{0}}{D(w, z)}\right]^{2 n}}
$$

where $D_{0}$ denotes the cutoff frequency, $D(w, z)$ the distance from the point $(w, z)$ to the image center $(M / 2, N / 2)$, and $n$ the filter order.

(3) After the filtering, the obtained image $G(w, z)$ is anticipated to be transformed back to the spatial domain through the inverse Fourier transform, i.e.,

$$
\Phi_{d e f}^{\prime}(x, y)=\sum_{w=0}^{M-1} \sum_{z=0}^{N-1} G(w, z) e^{j 2 \pi(w x / M+z y / N)}
$$

where $\Phi_{d e f}^{\prime}(x, y)$ represents the filtered deformed image in the spatial domain.

The above procedure summarizes only the main steps of the Butterworth filtering applied to IR images. Since the designed Butterworth filter is a kind of high-pass filter, a fundamental part of the thermal signal with low frequency characteristics may be filtered out simultaneously (in an unexpected way) during the filtering. Its quantity is supposed to be close to the mean value of $\Phi_{r e f}$, and this part should be compensated after the filtering. In this

way, the final obtained filtered function $\Phi_{d e f}^{\prime}(x, y)$ will be approaching to $\Phi_{r e f}$ (or $\left.\Phi_{i n i}\right)$, such that the goal of the filtering to eliminate the variation part $\Phi_{\text {var }}$ can be possibly achieved. In this first solution proposed, it is expected that the DIC is feasible even using the NCC criterion applied to the IR images after filtering.

\subsection{The second solution: advanced correlation criterion}

In this work, an advanced correlation criterion, mutual information (MI) [45], is employed instead of the NCC criterion for the purpose of a better handle on the image correlation problem under the unfavorable conditions as encountered in the IR-DIC implementation. The MI method can be used to evaluate the degree of correlation between two images via the measurement on the statistical dependence between the image intensities of corresponding pixels in both images. This method was originated in the information theory, and now has been applied successfully to the image correlation problems of deformable bodies [46-48]. It is adopted to treat the IR images in the present study. 
Regarding two IR images involved in the DIC, the image intensity values (i.e., radiative flux values) $a$ and $b$ of a pair of corresponding pixels in the two images can be registered to be two discrete random variables $A$ and $B$, respectively. The marginal probability distributions of the two variables are denoted by $p_{A}(a)$ and $p_{B}(b)$, respectively, and their joint probability distribution by $p_{A B}(a, b)$. These probability distribution functions can be estimated by simple normalization of the marginal and joint histograms of the overlapping parts of both images [48]. In this analysis, an important concept used in MI, the information entropy, is necessary to be introduced. The information entropy $H$ is defined to be a measure of the amount of uncertainty about a random variable. Here two entropies $H(A)$ and $H(B)$ for the two respective random variables $A$ and $B$ can be defined by the following equations

$$
\begin{aligned}
& H\left(A=-\sum_{a} p_{A}(a) \log p_{A}(a)\right. \\
& H(B)=-\sum_{b} p_{B}(b) \log p_{B}(b)
\end{aligned}
$$

with their joint entropy $H(A, B)$ defined as

$$
H\left(A, B=-\sum_{a, b} p_{A B}(a, b) \log p_{A B}(a, b)\right.
$$

In information theory, the MI of two random variables is a measure of the mutual dependence between the two variables. In essence, it quantifies the "amount of information" that can be obtained about one random variable through the other random variable. Generally, the MI of two discrete random variables $A$ and $B$ can be defined as

$$
I(A, B)=H(A)+H(B)-H(A, B)
$$

Substituting Eqs. (14)-(16) into Eq. (17), then we can obtain

$$
I(A, B)=\sum_{a, b} p_{A B}(a, b) \log \left(\frac{p_{A B}(a, b)}{p_{A}(a) p_{B}(b)}\right)
$$

Concerning the specific application in the DIC, one of the two random variables (e.g., $A$ ) is always fixed as the reference image, i.e., $\Phi_{r e f}(x)$, and the other random variable (e.g., $\left.B\right)$ is changeable through the transformation $T_{\mu}$ as the transformed image, i.e., $\Phi_{d e f}[R(x)]$. In the process of image registration, an optimizer is necessary to be designed in order to search the optimal $B$ that shares the most mutual dependence with $A$. The objective of the optimization is to maximize the MI function $I(A, B)$ that represents the reduction in the uncertainty of one 
random variable by the knowledge of the other. When a maximal $I(A, B)$ is achieved, it means that the two images $\Phi_{r e f}(x)$ and $\Phi_{d e f}[R(x)]$ are best spatially overlapped.

The advantage of the MI criterion is that it relies on the probability distributions of the image intensities but not their absolution values, such that no particular assumptions, e.g., linearity, are imposed in the relationship between the two images. It is, therefore, more general and powerful than the NCC criterion. In this work, the MI criterion is expected to be able to realize the DIC based on the thermal images of deforming material, which are partly dependent on the surface temperature evolution.

\subsection{Lagrangian thermography}

The untreated experimental temperature field is described in the Euler coordinate system as acquired by an IR camera at a fixed position. A drawback of this description is that the temperature evolution of a given material point cannot be followed owing to the unknown trajectory of the point during deformation. This problem can be schematically illustrated by Fig. 1. The temperature of a material point $\mathrm{P}$ evolves from $T_{0}$ to $T_{1}$ from the moment $t_{0}$ to $t_{1}$, and in the meanwhile this point is displaced from location A to B in the Euler coordinate system. The temperature of location A at moment $t_{1}$ is $T_{2}$, thus the recorded temperature variation of point $\mathrm{P}$ will be $T_{2}-T_{0}$ but not the true temperature variation of the material point $\mathrm{P}$ that should be $T_{1}-T_{0}$. Furthermore, a parasitic error can be also caused in this process. Since the movement of the material point cannot be tracked, its emissivity cannot be tracked as well. In this circumstance, the temperature estimation based on the initial emissivity map in the undeformed state will become unreliable. Hence, it is of great interest to transform the Euler coordinate system into Lagrangian coordinate system. The only requirement of this transformation is the obtainment of the Lagrangian displacement field, which is accessible by the IR-DIC. 

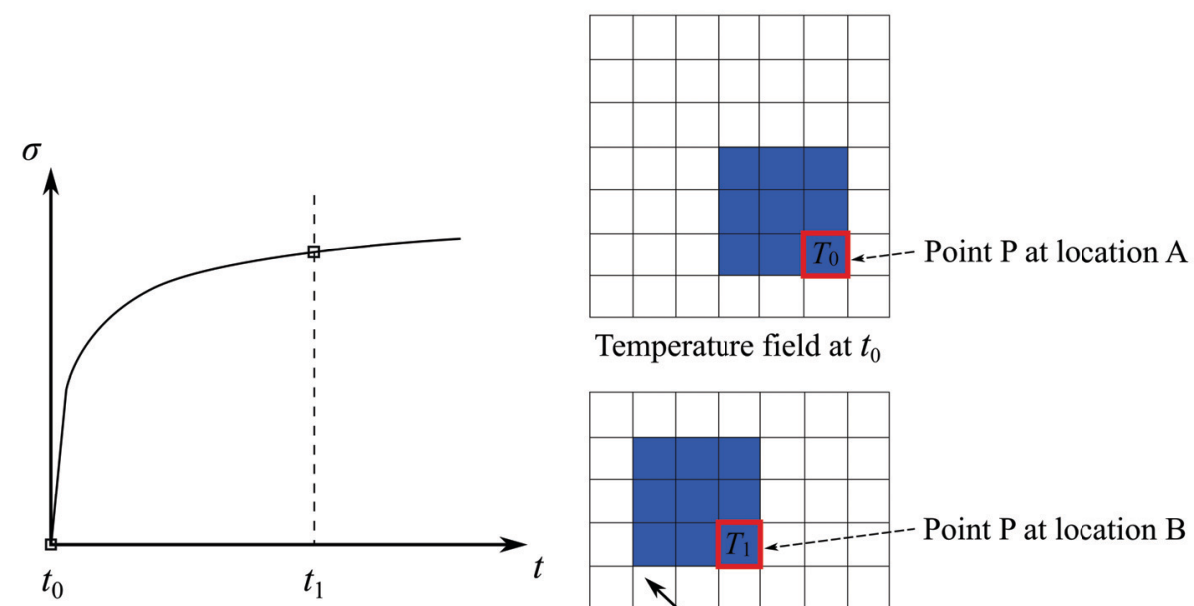

Temperature field at $t_{0}$

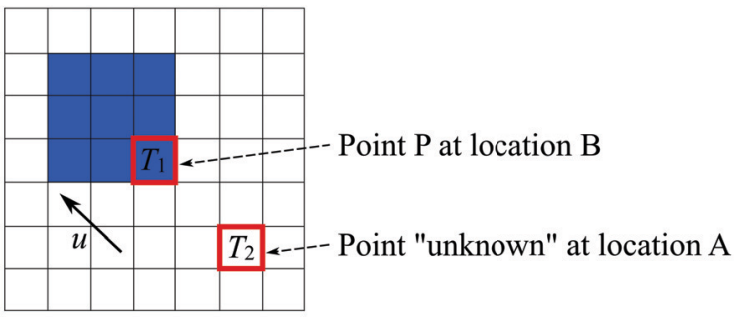

Temperature field at $t_{1}$

Fig. 1. A schematic presentation of the Lagrangian thermography in describing the temperature field evolution of specimen surface during deformation

Thanks to the image correlation method, the thermal fields $\Phi_{d e f}(x)$ of specimen surface during deformation can be, one by one, transformed into the reference coordinate system, i.e., $\Phi_{d e f}[R(x)]$ can be estimated. With the known initial emissivity map of specimen surface, it allows evaluating the temperature of an arbitrary material point at pixel $x$ for a given moment $t$ by the following equation according to Eq. (8):

$$
T(x, t)=\left(\frac{\left.\Phi_{d e f}[R(x, t), t]\right)}{\sigma \varepsilon(x)}\right)^{1 / 4}
$$

The emissivity field of the specimen surface should be measured before the mechanical testing, i.e., in the undeformed state. And an in-situ emissivity measurement is necessary, such that the evaluated emissivity field is applicable to the thermal field measured in the mechanical test.

\section{Experiment}

The proposed methods are necessary to be validated in the mechanical testing. To this end, a special experiment was conducted by using a notched specimen stretched in a tensile test. It is a flat specimen with a penetrated round hole in the specimen center, with the geometrical dimensions as shown in Fig. 2. In the tensile test, the central hole will lead to important stress 
concentrations at the adjacent regions of the hole boundary where relatively high and inhomogeneous deformations can be produced. In this circumstance, the Euler thermography may become incompetent in following the thermal evolution of the material points near the hole boundary because of the high and inhomogeneous deformation, and consequently a Lagrangian field description is expected. Hence, by examining the thermal field evolution near the hole boundary can provide an effective way to verify the performance of the proposed approach in realizing a proper presentation of the Lagrangian thermal field. It is anticipated to be compared with the untreated Euler thermal field presentation. Moreover, a verification on the kinematic field evolution by the IR-DIC is also necessary, which is the prerequisite of the correct implementation of the Lagrangian thermography.

The material of the test specimen was DP 590, as a typical dual phase steel mostly used in automobiles. Its ultimate tensile strength was about $595 \mathrm{MPa}$ and elongation to failure about $35 \%$ as determined in a tensile test. A speckled coating was applied to the specimen surface before the mechanical test. It was realized by a high-emissivity matte black paint partially sprayed on the raw specimen surface with relatively low emissivity, resulting, therefore, a heterogeneous emissivity distribution. The emissivity values of the neighboring points on the specimen surface are in good contrast due to the nature of the speckled coating. This manmade coating allows reflecting a speckled pattern effect in the acquired thermal fields (expressed in radiative flux) of specimen surface that could facilitate the DIC calculation based on IR images.

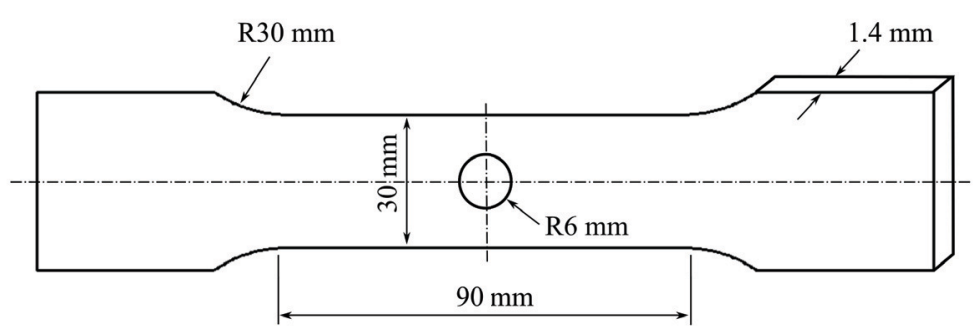

Fig. 2. The geometrical dimensions of the notched tensile specimen

A uniaxial tensile test was performed at the room temperature and at a constant cross-head velocity of $0.025 \mathrm{~mm} / \mathrm{s}$ using an electromechanical testing machine Instron 5984 . The test was suspended until the rupture of specimen. During the mechanical loading, the deforming 
specimen surface was filmed by a high-performance IR camera FLIR SC7700M at a sampling frequency of $20 \mathrm{~Hz}$. It allowed providing an image size of $512 \times 640$ pixels and physical resolution of $0.156 \times 0.156 \mathrm{~mm}^{2}$ per pixel in the present experimental set-up. The IR camera was calibrated in advance using an extended black body. The employed calibration method was a so-called pixel-to-pixel calibration, which meant that each sensor in the IR focal plane array (FPA) was calibrated one by one in a single calibration experiment [7]. In this way, the acquired raw thermal signal DL can be converted into radiative flux $\Phi$ through the calibration curve of each IR sensor of the FPA.

Fig. 3 shows a schematic presentation of the experimental set-up of the thermographic measurement during the tensile test. The IR camera was positioned in the front of the specimen surface for imaging the thermal field evolution. In addition, a massive cold source made of ice was also placed in front of the specimen surface. Its function was to diminish the radiative flux emitted by the environment, thus the acquired thermal data can be more inherent to the material behavior with little environmental influence.

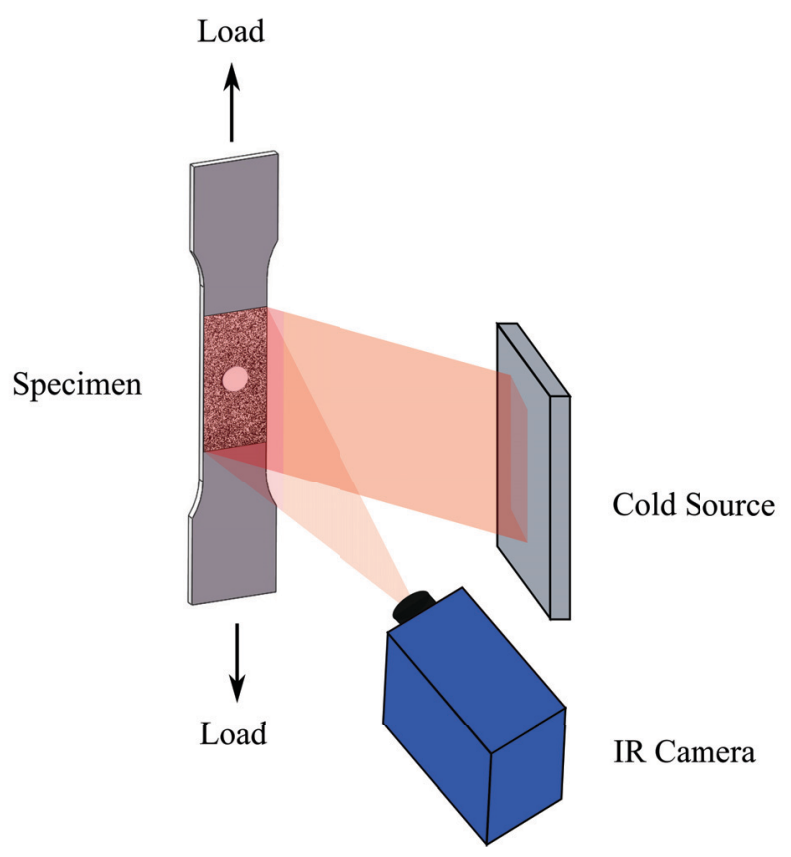

Fig. 3.Schematic presentation of the experimental set-up of the thermographic measurement in the tensile test 
The objective of the experiment is to realize simultaneous assessment of Lagrangian strain and temperature fields by IR-DIC. To this end, two technical solutions have been proposed for facilitating the DIC computation based on IR images, as presented previously. Then, the IR-DIC allows the assessment of the kinematic fields (i.e., the displacement and strain fields), with the knowledge of which the transformation of the thermal field from Euler coordinate system to Lagrangian coordinate system is straightforward. Hence, a first verification on the experimental results should be focused on the evaluated kinematic fields. And then further verification on the Lagrangian thermal field evolution is necessary to be conducted. The results and discussion of the two aspects are presented in the following section.

\section{Results and discussion}

\subsection{Kinematic field assessment}

The kinematic field assessment of the notched specimen during the tensile test can be realized independently by the two solutions proposed, and the obtained results are discussed singly.

\subsubsection{Results of the first solution}

The feasibility of the first solution based on a high-pass filtering was verified as the first step. The designed Butterworth filter was applied to each acquired image of specimen surface in the deformed state, and then correlations were performed, one by one, with respect to the reference image using the NCC criterion. A representative example is illustrated by Fig. 4 to
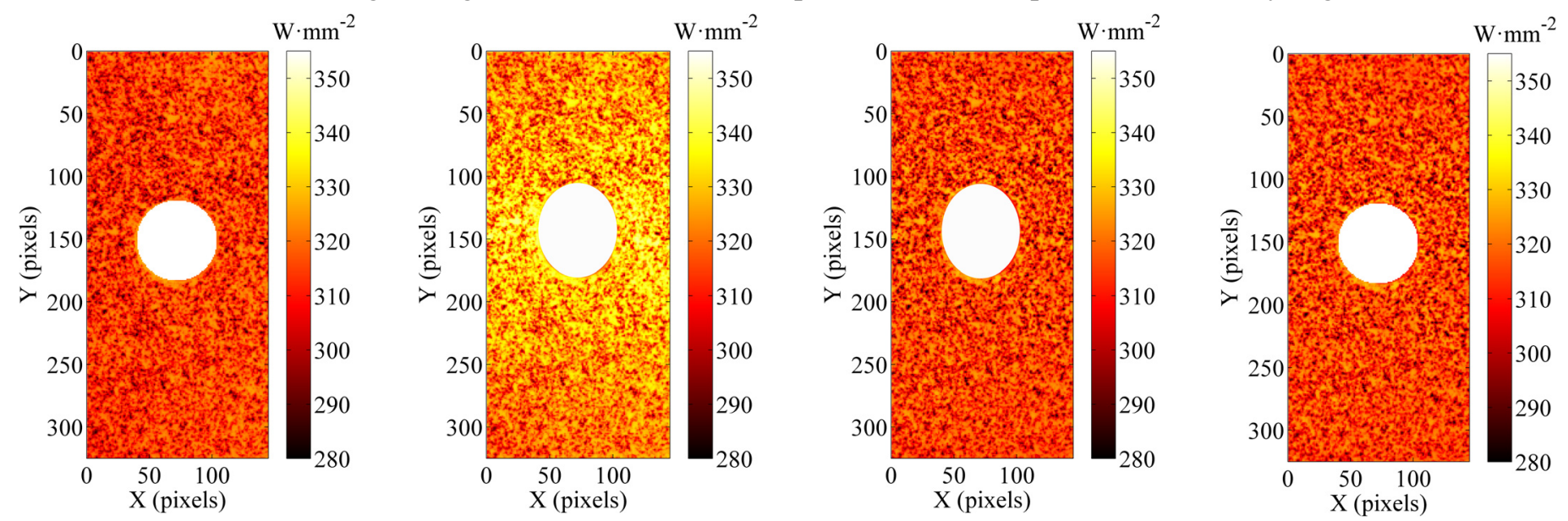

demonstrate the effect of the image filtering. Fig. 4(a) shows the reference image expressed in 
radiative flux in the undeformed state, and Fig. 4(b) displays a deformed image at a given moment (120 s) during the tensile test. It can be remarked in Fig. 4(b) that the central round hole is stretched in the axial direction and an important thermal evolution manifests on the specimen surface. The thermal intensity is enhanced on the whole, and it tends to be more concentrated near the hole boundary comparing to the initial undeformed state with a relatively homogeneous distribution. In light of the great variation of thermal signal from Fig. 4(a) to Fig. 4(b), a direct image correlation between the two images based on the NCC criterion could be problematic. Since the variation part of the thermal signal demonstrates as a low frequency signal, a high-pass Butterworth filter was applied to Fig. 4(b), resulting in a filtered deformed image shown in Fig. 4(c). In the designed Butterworth filter, the filter order was set as a fixed value equal to 2 , and the cutoff frequency $D_{0}$ was a variable depending on the current thermal signal intensity. Its basic value was set as 2 that should be increased continuously during the deformation process with the intensification of the thermal signal. After the high-pass filtering, one can note in Fig. 4(c) that the variation part of radiative flux has been eliminated to a certain degree and the apparent image pattern becomes similar to the reference image. At this stage, the filtered image was considered appropriate, and it was correlated to the reference image by the IR-DIC algorithm using the NCC criterion. It led to a transformed image shown in Fig. 4(d). A good geometrical similarity can be noted between Fig. 4(a) and Fig. 4(d), which indicates a satisfactory image correlation being executed.

Fig. 4. A representative example of the implementation of the first solution: (a) the reference image, (b) a selected deformed image at $120 \mathrm{~s}$, (c) the deformed image after the high-pass filtering, (d) the transformed image by IR-DIC

A further verification on the image filtering effect was conducted by evaluating the image histogram. Concerning the IR images, the image histogram here refers to the plot of the number of pixels as a function of radiative flux. Fig. 5(a) shows the comparison between the image histogram of the reference image in Fig. 4(a) and that of the unfiltered deformed image 
in Fig. 4(b). A distinct difference can be observed between the two image histograms. It is apparently contradictive to the hypothesis of the conservation of optical flow. After applying the image filtering, the image histogram of the filtered deformed image was compared again with that of the reference image, as shown in Fig. 5(b). The histograms of the two images are basically overlapped that indicates a satisfactory similarity of the thermal distributions in the two images. Hence, it provides a favorable condition for the IR-DIC implementation under the "conservation of optical flow".

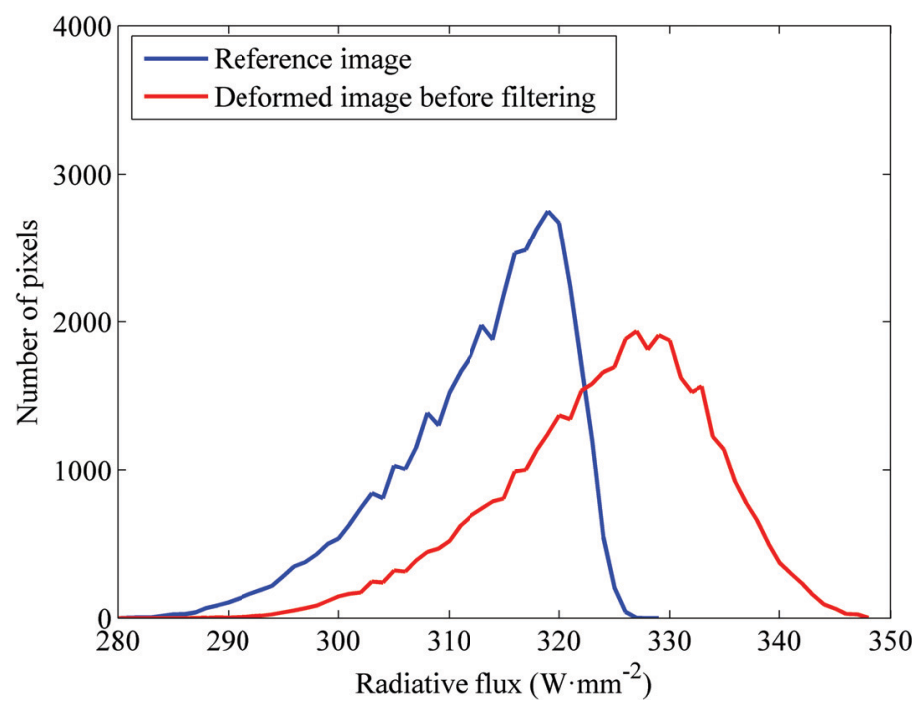

(a)

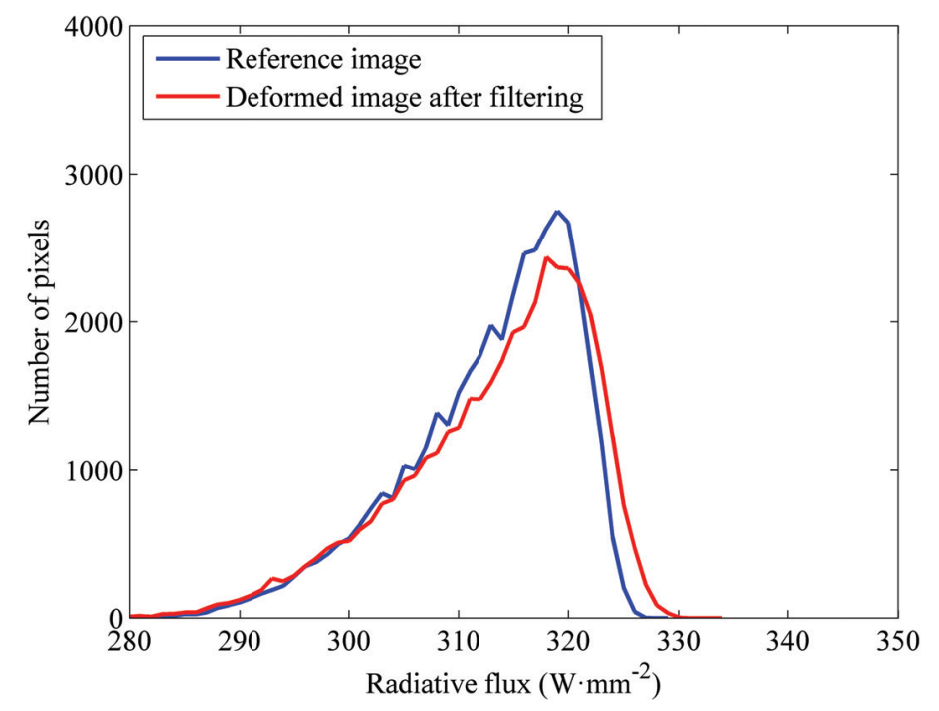

(b)

Fig. 5. (a) Comparison between the image histograms of the reference image and the unfiltered deformed image at $120 \mathrm{~s}$; (b) comparison between the image histograms of the reference image and the filtered deformed image at $120 \mathrm{~s}$

The quality of the image correlation is necessary to be verified. Generally, the error of image correlation can be measured by the residual image (or correlation residual) [1] that is defined as the difference between the reference image and transformed image. In the present work, the residual image is denoted by $\Phi_{\text {res }}(x)$, which can be evaluated by

$$
\Phi_{r e s}(x)=\Phi_{r e f}(x)-\Phi_{\text {def }}[R(x)]
$$

For each transformed image, its corresponding residual image was calculated through the above equation. Fig. 6 illustrates the load-strain curve of the specimen during the tensile test, in which a sequence of residual images at the selected moments (points A-E) is displayed. In 
general, the absolute errors of the DIC calculation remain on a low level with average values approaching to zero. More importantly, there are no particular errors with well-defined geometries appeared in the residual images, which implies that the error distributions are also reasonable. A notable evidence is that the central hole is still round even at greater deformations in the residual images (expressed in the Lagrangian coordinate system), and there are no shadows (non-overlapped areas) appeared at the hole boundary. The latter is normally considered as the marker of the DIC error.

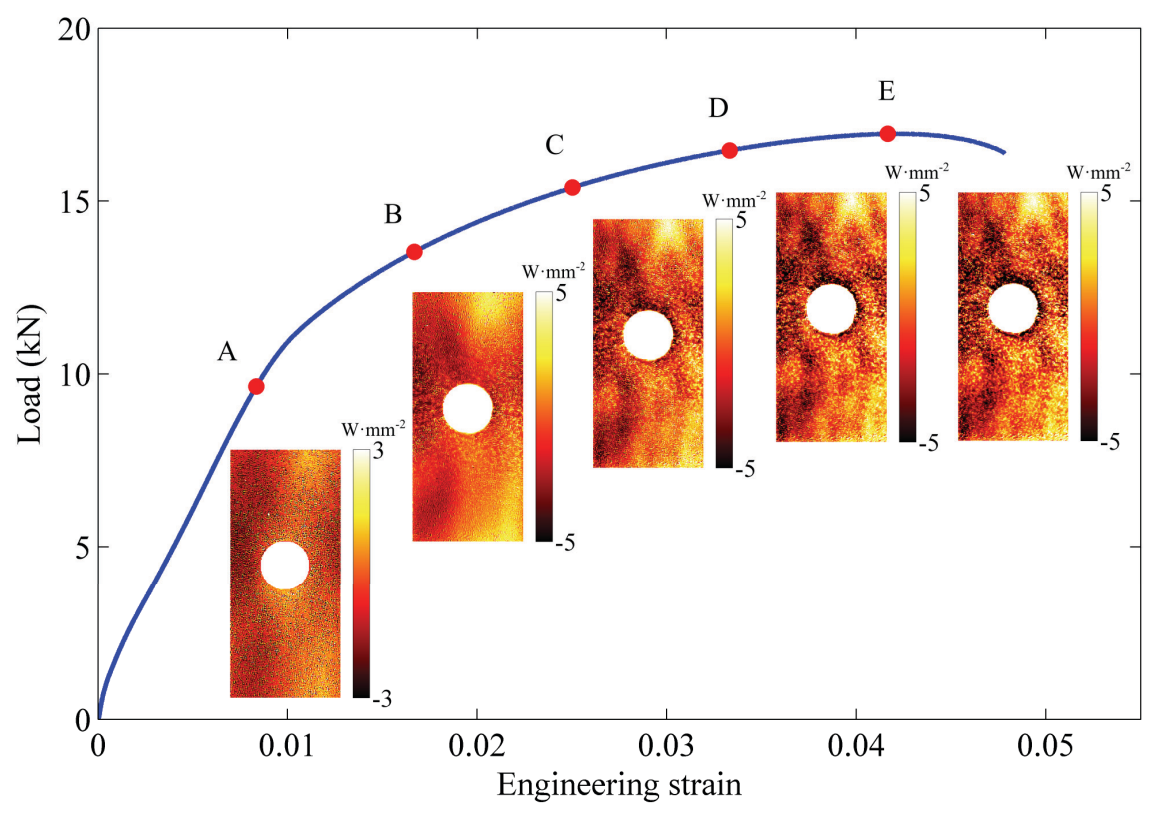

Fig.6. A sequence of evaluated residual images at the selected moments during the tensile test obtained by the IR-DIC based on the NCC criterion

From the DIC calculation based on IR images of specimen surface, the displacement and strain fields for each moment of image acquisition can be estimated. The displacement field is the direct output of the DIC computation, and then the strain field can be calculated by differentiation using, for instance, the finite difference method. Fig. 7 illustrates a series of the axial displacement fields at the different deformation stages during the tensile test. It shows that the estimated displacements are symmetric with respect to the middle line of the specimen along the vertical direction. And the displacement variation near the hole boundary is seemingly more important than the regions far away from the hole. For the ease of interpretation, the corresponding axial strain fields of the illustrated displacement fields are 
shown in Fig. 8. The estimated strain fields demonstrate well the strain localization effect near the central hole boundary as the consequence of the stress concentration. This effect tends to be more marked with the increment of macroscopic deformation. To verify the correctness of the strain distributions obtained by the IR-DIC, the numerical simulation of the tensile deformation process of the notched specimen was performed using the commercial FEM software ABAQUS. Fig. 9 shows a series of simulated axial strain fields at the different deformation stages. It can be remarked that the displayed strain patterns by simulation are rather similar to that estimated by the DIC measurement as shown in Fig. 8. It, therefore, validates the effectiveness of the proposed method.

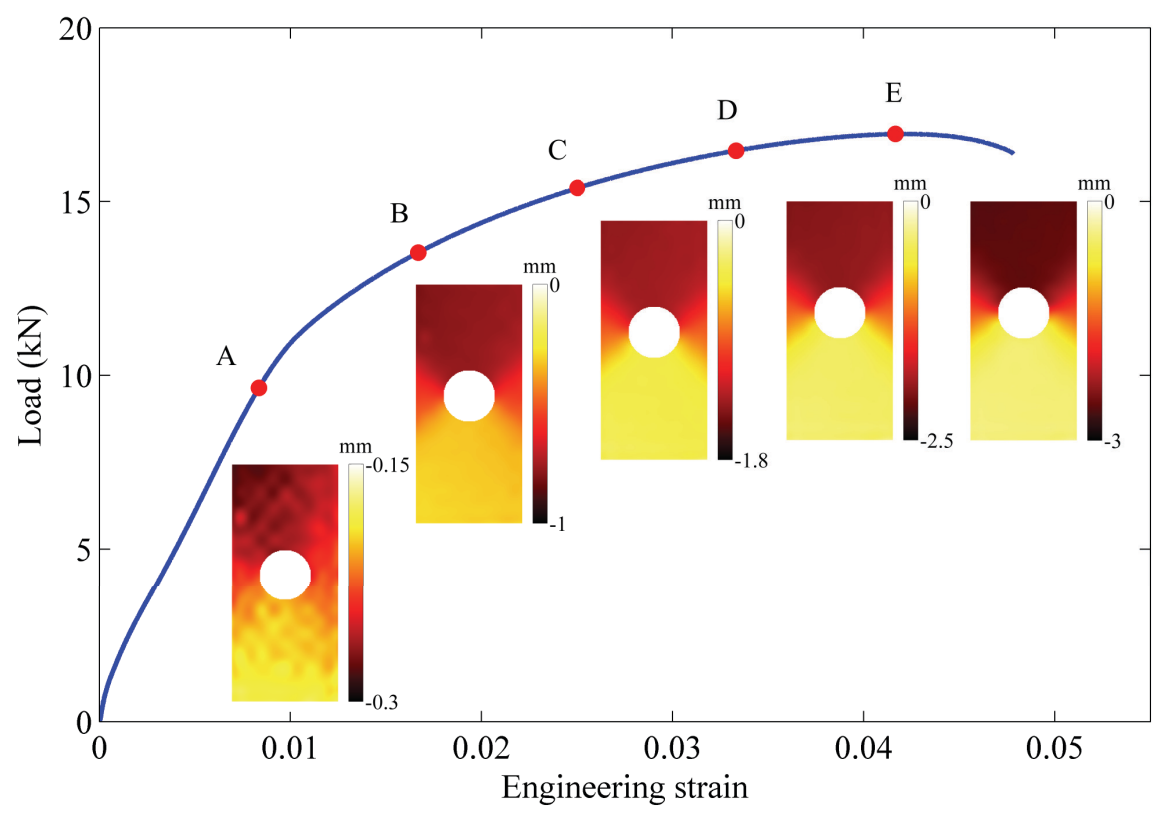

Fig.7. A series of selected axial displacement fields during the tensile test obtained by the IR-

DIC based on the NCC criterion 


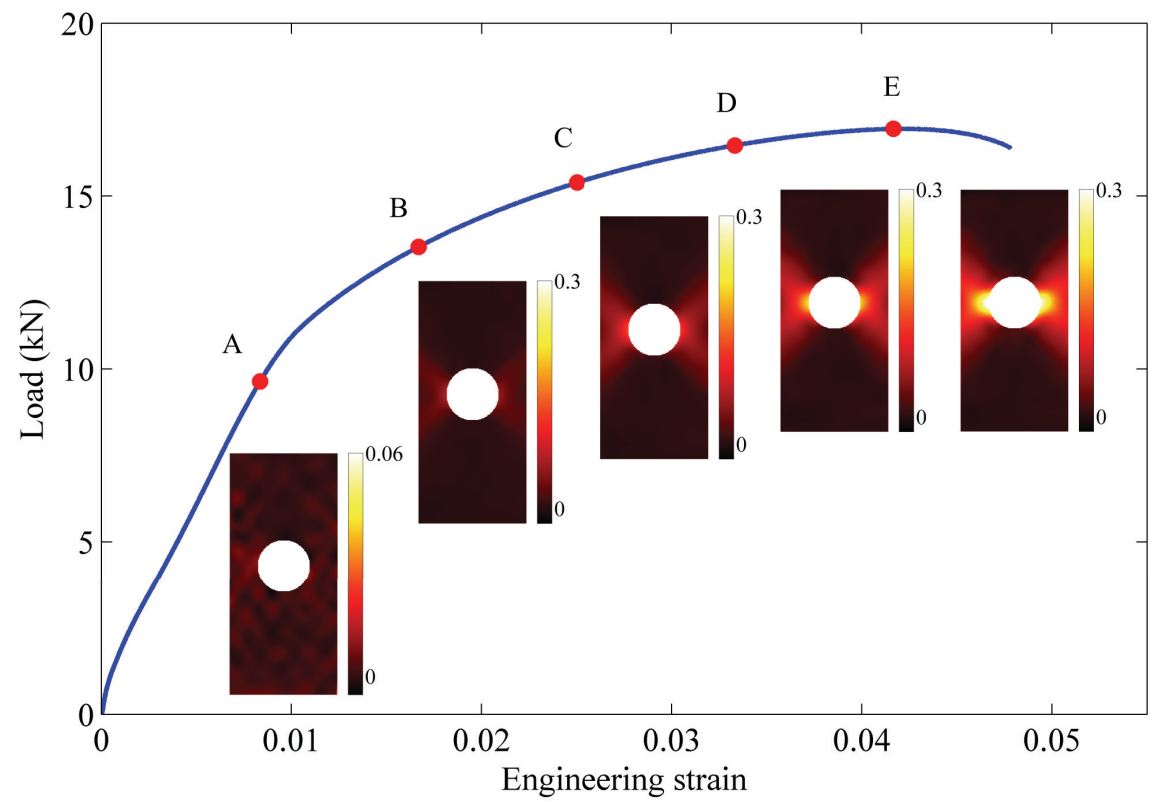

Fig.8. A series of selected axial strain fields during the tensile test obtained by the IR-DIC based on the NCC criterion
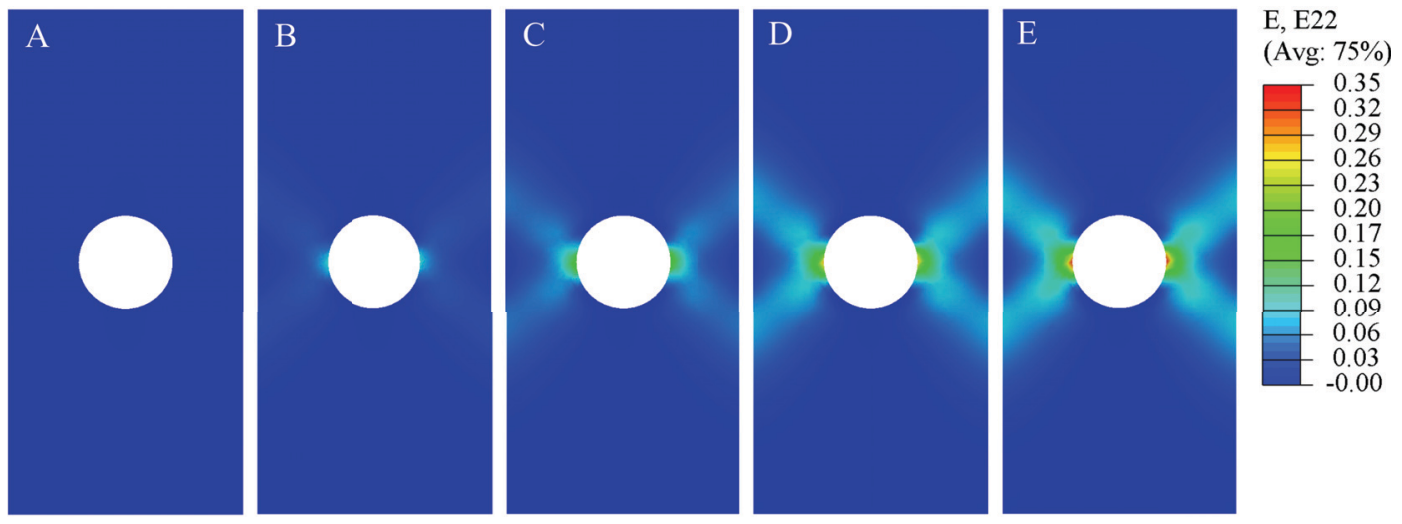

Fig.9. A series of selected axial strain fields during tensile deformation obtained by the FEM

\subsubsection{Results of the second solution}

The core of the second solution is the adaptation of the correlation criterion by using the MI instead of the NCC. It does not need any special treatment on the deformed images, but instead it utilizes the raw acquired images for the DIC calculation. Hence, the implementation of the second solution is more straightforward than the first solution. According to this method, the similarity of the reference image and deformed image is measured by their mutual information. A best transformation function is searched by the optimization algorithm with the objective to maximize the similarity between the two images. Then it allows providing the displacement and strain fields. The precision of the DIC calculation can still be 
evaluated by the residual image. Fig. 10 shows a sequence of the evaluated residual images at the selected moments (identical to Fig. 6) during the tensile test. The reasonability of the results embody that the shape of the round hole is unchanged during the deformation and no particular shadows appear at the hole boundary. Meanwhile, it can also be noted that the absolute values of the obtained correlation residuals are generally greater than that given by the first solution by comparing Fig. 10 with Fig. 6 . It is mainly due to the fact that the deformed IR images are unfiltered in the present DIC strategy, thus the variation part of the thermal signal $\Phi_{\text {var }}$ may remain in the residual images. Nevertheless, it should be emphasized that the quality of the DIC is not measured by the absolute values of the correlation residual but by its distribution, which actually reflects the spatial overlapping degree of the two images (reference image and transformed image). For the present case, the roundness of the central hole and as well as the sharpness of the hole boundary can be considered as the evaluation criterion of the DIC quality.

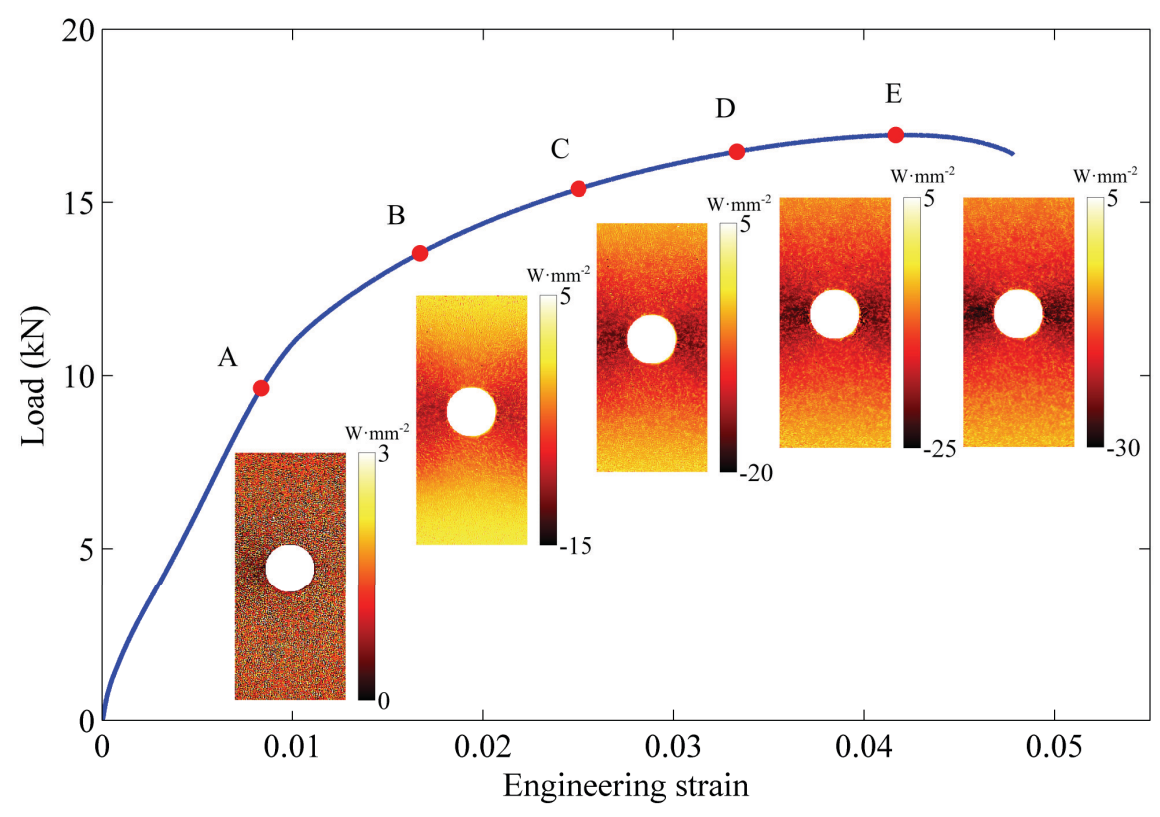

Fig.10. A sequence of evaluated residual images at the selected moments during the tensile test obtained by the IR-DIC based on the MI criterion

Furthermore, the performance of the DIC strategy based on the MI criterion can be directly verified by examining the resulting kinematic fields. A sequence of the axial displacement and strain fields at the selected moments (points A-E) during the tensile test are 
shown in Fig. 11 and Fig. 12, respectively. By comparing to Fig. 7 and Fig. 8, one can note that the evaluated kinematic fields by the present method are rather comparable to that provided by the first solution, not only in the absolute values but also in the distribution characteristic. Hence, the performance of the second solution is considered as desirable as the first solution.

After obtaining the kinematic fields, the assessment of the temperature evolution of the material points is possible. It is discussed in the following section.

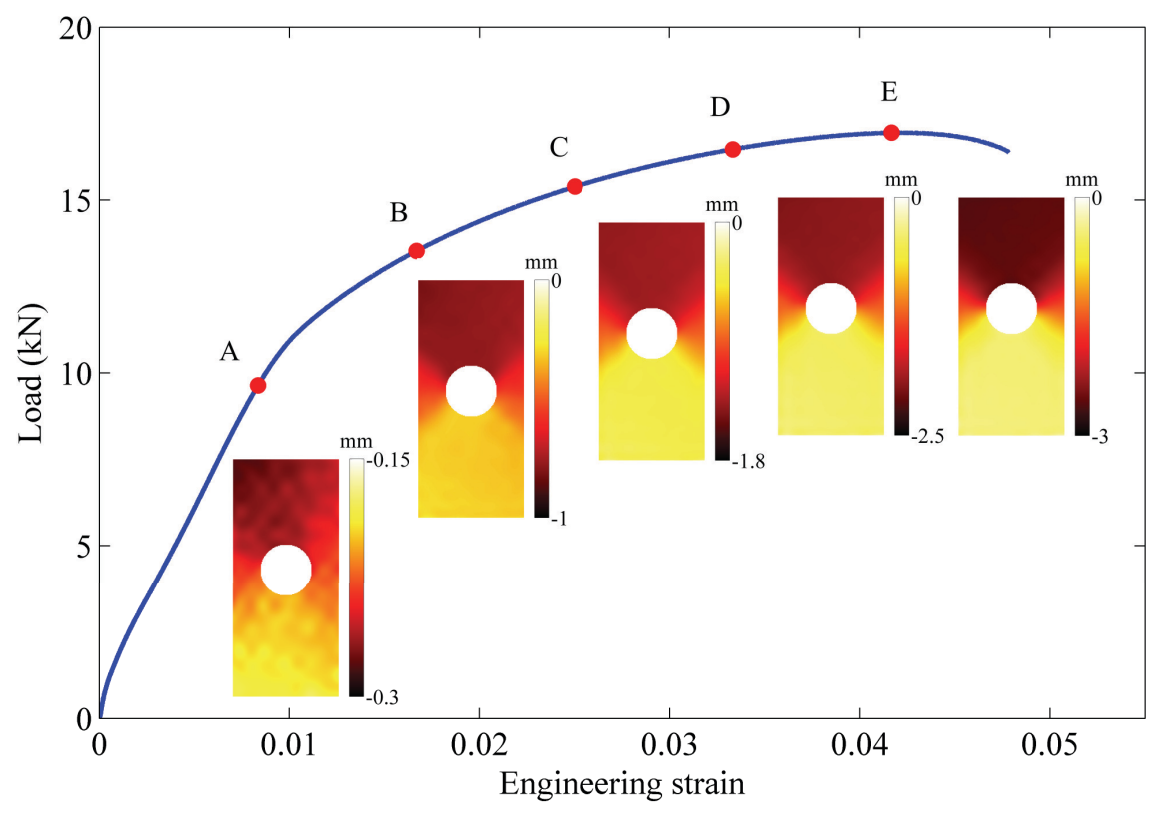

Fig.11. A series of selected axial displacement fields during the tensile test obtained by the IR-DIC based on the MI criterion 


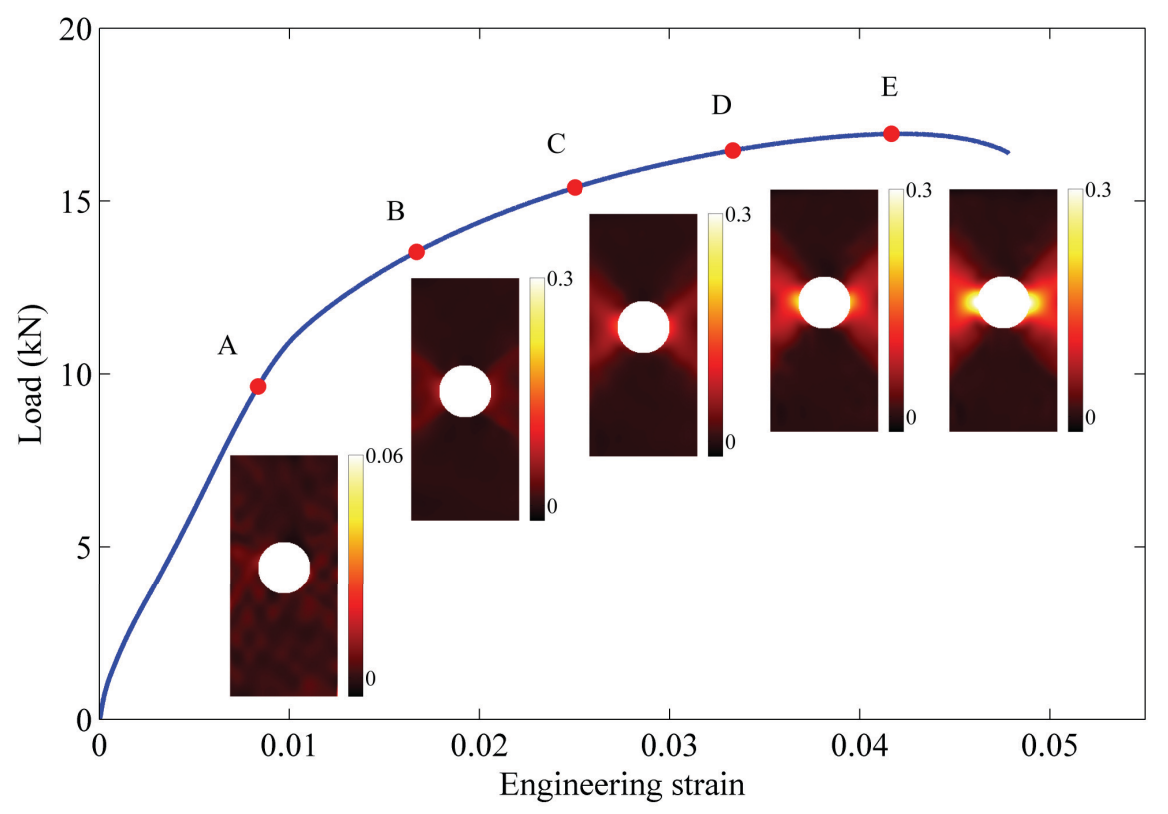

Fig.12. A series of selected axial strain fields during the tensile test obtained by the IR-DIC based on the MI criterion

\subsection{Lagrangian thermal field evolution}

Thanks to the proposed IR-DIC method, the transformed thermal images are described in the Lagrangian coordinate system. It allows therefore following the thermal evolution of the material points of specimen surface during the deformation. The temperature variation (or relative temperature) of an arbitrary pixel $x$ in the thermal image can be estimated by

$$
\Delta T(x, t)=T(x, t)-T_{0}(x)
$$

where $T_{0}(x)$ presents the initial temperature in the undeformed state.

In this analysis, the thermal evolution is characterized using the relative temperature term. Fig. 13 illustrates the Lagrangian temperature field evolution of specimen surface during the tensile test. In the early stage of the tensile loading, the deformation is linear elastic, thus the temperature of specimen surface manifests a slight decrease due to thermoelasticity, as shown by the negative values in the temperature field A in Fig. 13. With the increase of load, the temperature rises gradually and globally due to macroscopic plastic deformation accumulation. And the most important temperature increment occurs in the areas near the central hole, especially for the regions along the horizontal direction subjected to higher tensile stresses. The maximum temperature increment is about $6{ }^{\circ} \mathrm{C}$ during the tensile test. 


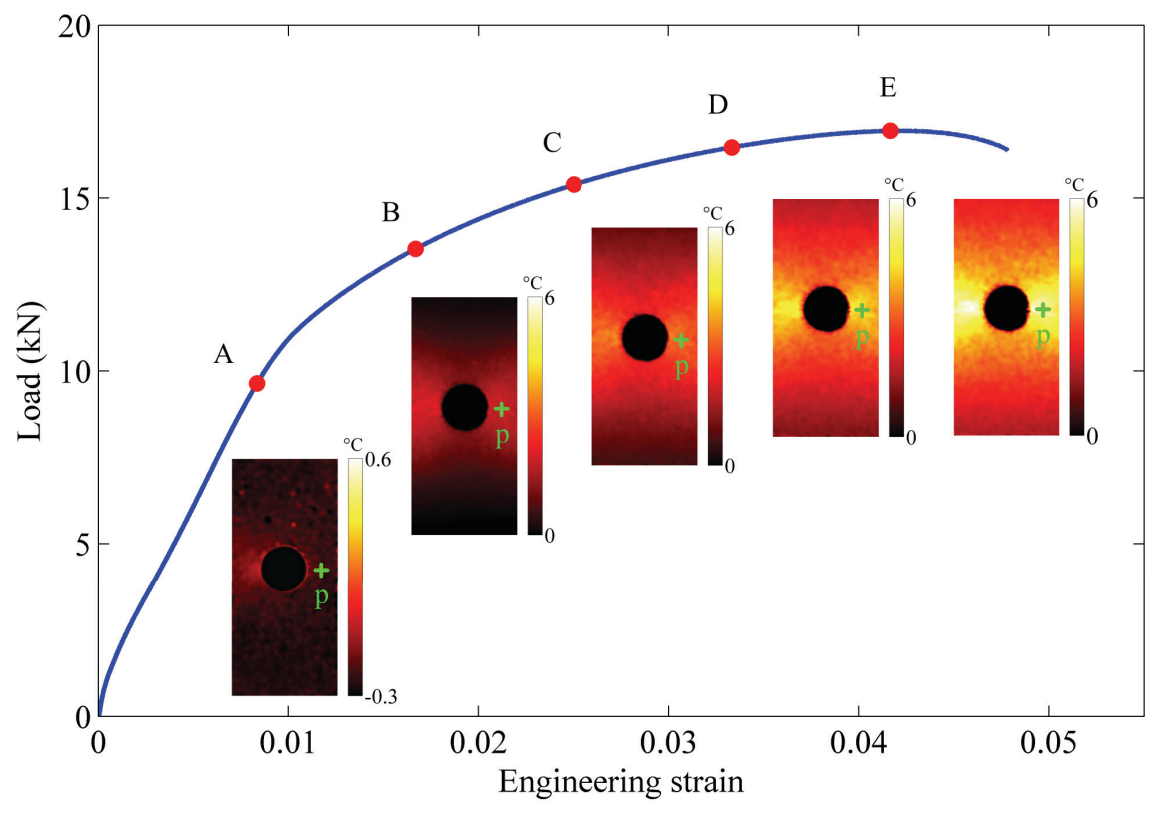

Fig.13. The Lagrangian temperature field evolution during the tensile test

For the purpose of comparison, the raw Euler thermal field evolution is also presented. Fig. 14 illustrates a sequence of the Euler temperature fields during deformation at the same moments with the Lagrangian temperature fields in Fig. 13. One can note that widespread noises present in the Euler temperature fields through the whole deformation process. Here the Euler temperature calculation is based on the initial emissivity estimate in the undeformed state. It is, however, incorrect, as the material points are motive during the deformation. In other words, the emissivity of a fixed pixel in the thermal image should be variable during the deformation as different material points will pass through this pixel in this process. For the present case, in the initial emissivity configuration the difference in emissivity between the neighboring material points is significant, thus the error of emissivity estimate by using a fixed initial value during the deformation process may become important. For a better illustration, the temperature evolution of a fixed pixel in the thermal image is characterized by the Lagrangian thermography and Euler thermography, respectively. Their comparison is shown in Fig. 15. It shows that the Lagrangian temperature of the pixel demonstrates a reasonable evolution trend with monotonic increase induced by plastic strain accumulation, except the initial drop due to thermoelasticity. In contrast, the evolution of the Euler temperature appears irregular with the abnormal drops in the plastic deformation stage. 


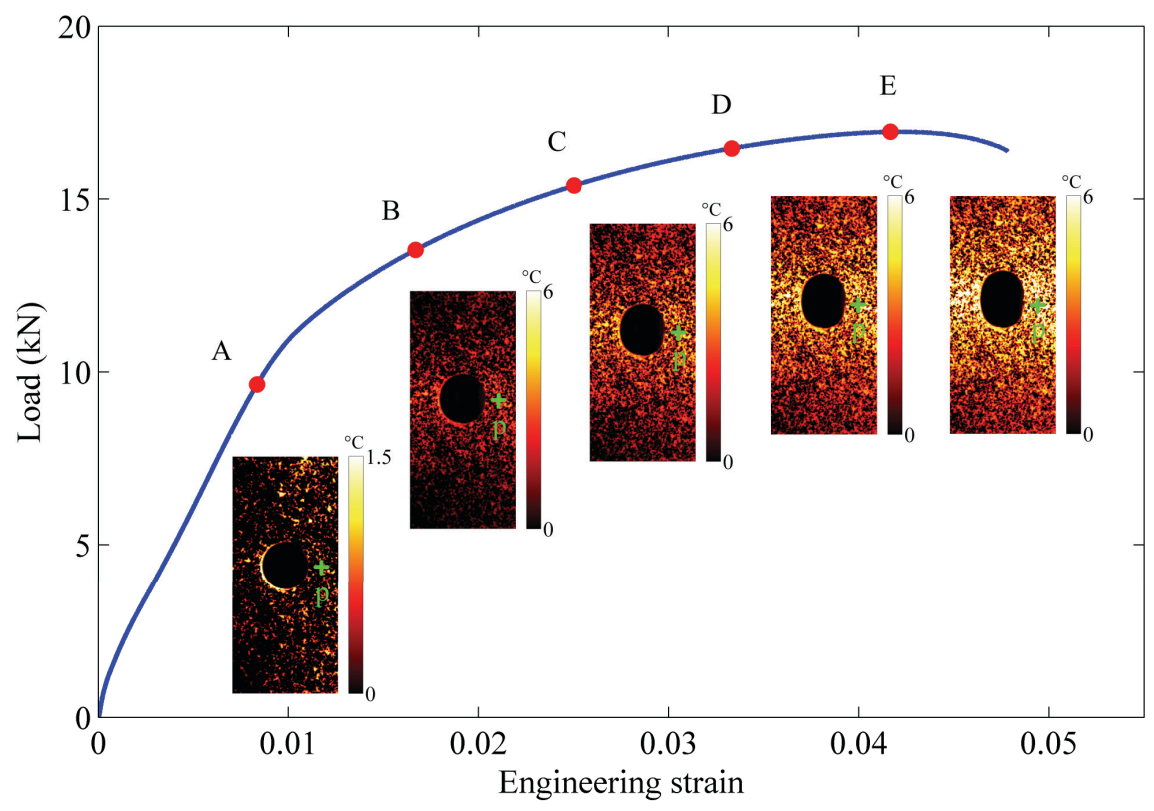

Fig.14. The Euler temperature field evolution during the tensile test

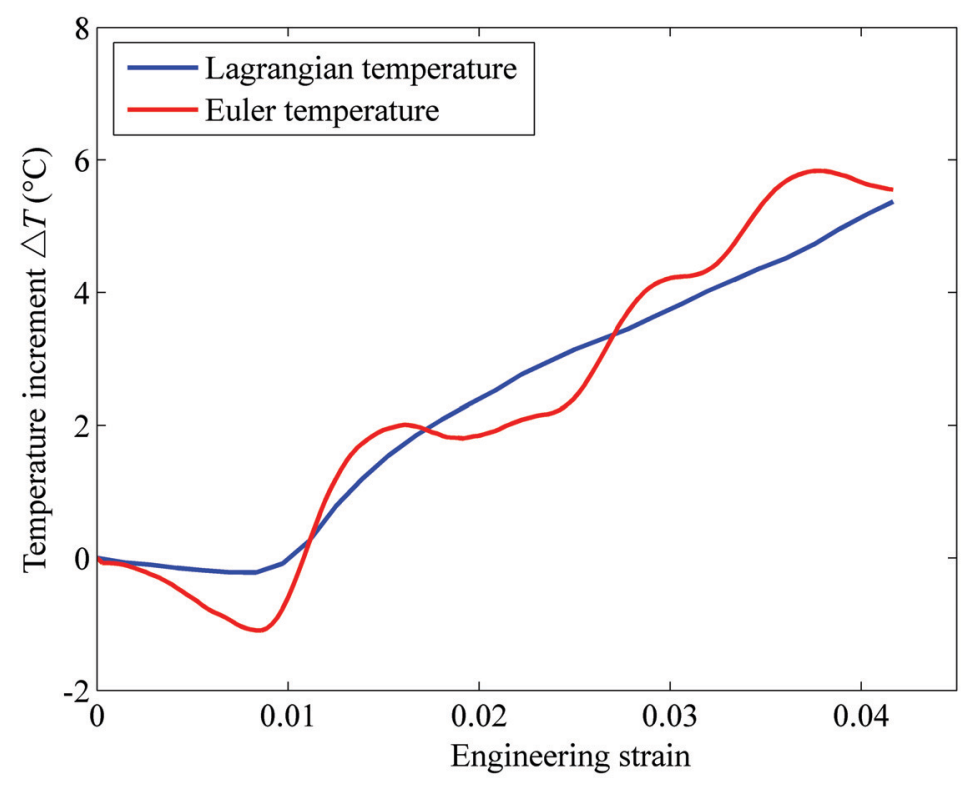

Fig.15. The comparison of the Lagrangian temperature and Euler temperature evolution of a fixed pixel in the thermal image during the tensile test

The present example demonstrates well the defect of the Euler thermography and as well as the practical utility of the Lagrangian thermography. It is remarked that the Lagrangian thermography is particularly useful for the cases of high deformation (or strong strain localization) and non-uniform emissivity distribution on the material surface.

\section{Conclusion}


An improved IR-DIC strategy for simultaneous assessment of Lagrangian strain and temperature fields has been developed in this paper. A specialty of the IR-DIC applied to solid deformation is that the temperature variation of material surface may bring certain difficulties to the image correlation as the hypothesis of the conservation of optical flow may become relative in such circumstance. To solve this problem, two solutions were proposed in this work. In the first solution, a high-pass filter was designed in order to eliminate the unexpected variation of the thermal signal, thus the DIC calculation could work even using a classical NCC criterion. In the second solution, a more general and powerful metric, MI, was adopted as the correlation criterion for facilitating the DIC calculation even under the unfavorable conditions. Both of the two solutions were verified in the experiment. It showed that both solutions enabled providing the kinematic field assessment in acceptable precision, and then the transformation from Euler to Lagrangian coordinate system was feasible. Thanks to the Lagrangian thermal filed presentation, the temperature evolution of a given material point can be followed effectively during the deformation.

The developed method needs only a single IR camera for actualizing the simultaneous measurement of Lagrangian strain and temperature fields. It provides, therefore, a relatively simple and economic solution comparing to the two-camera set-up. The obtained Lagrangian temperature field is demonstrated to be useful in describing a high and/or inhomogeneous deformation process. Potentially, it could be applied to characterize the deformation of an elastomer or the heterogeneous strain field evolution near a crack tip.

\section{Acknowledgements}

This work is supported by the Major Program of National Science Foundation of China (No. 51490662), the National Science Foundation for Excellent Young Scholars of China (No. 51222502), the National Science Foundation of China (No. 51605153), the Fund for Distinguished Young Scientists of Hunan Province of China (No. 14JJ1016), and the Open Fund of Key Laboratory of Electronic Equipment Structure Design (Ministry of Education) in Xidian University (No. EESD-OF-201502).

\section{References}


[1] M. Grédiac, F. Hild, Full-field measurements and identification in solid mechanics, WileyISTE, 2012.

[2] M.A. Sutton, J.J. Orteu, H. Schreier, Image correlation for shape, motion and deformation measurements: basic concepts, theory and applications, Springer Science \& Business Media, 2009.

[3] B. Pan, K. Qian, H. Xie, A. Asundi, Two-dimensional digital image correlation for inplane displacement and strain measurement: a review, Measurement Science and Technology, 20 (2009) 062001.

[4] B. Wang, B. Pan, G. Lubineau, Some practical considerations in finite element-based digital image correlation, Optics and Lasers in Engineering, 73 (2015) 22-32.

[5] G. Gaussorgues, S. Chomet, Infrared thermography, Springer Science \& Business Media, 2012.

[6] A. Chrysochoos, Infrared thermography applied to the analysis of material behavior: a brief overview, Quantitative InfraRed Thermography Journal, 9 (2012) 193-208.

[7] X.G. Wang, J.F. Witz, A. El Bartali, A. Oudriss, C. Jiang, Quantitative infrared thermography applied to subgrain scale and the effect of out-of-plane deformation, Infrared Physics \& Technology, 71 (2015) 432-438.

[8] S. Guo, M. Sutton, N. Li, X. Li, L. Wang, S. Rajan, Measurement of Local Thermal Deformations in Heterogeneous Microstructures via SEM Imaging with Digital Image Correlation, Experimental Mechanics, (2016) 1-16.

[9] E. Lindfeldt, M. Ekh, K. Cvetskovski, M. Schilke, Using DIC to identify microscale strain fields from in-situ SEM images of a pearlitic steel, Experimental Mechanics, 54 (2014) 1503-1513.

[10] C.C. Tasan, J.P. Hoefnagels, M. Diehl, D. Yan, F. Roters, D. Raabe, Strain localization and damage in dual phase steels investigated by coupled in-situ deformation experiments and crystal plasticity simulations, International Journal of Plasticity, 63 (2014) 198-210.

[11] H. Louche, P. Vacher, R. Arrieux, Thermal observations associated with the Portevin-Le Châtelier effect in an Al-Mg alloy, Materials Science and Engineering: A, 404 (2005) 188-196.

[12] G. Besnard, F. Hild, S. Roux, "Finite-element" displacement fields analysis from digital images: application to Portevin-Le Châtelier bands, Experimental Mechanics, 46 (2006) 789-803.

[13] P.D. Zavattieri, V. Savic, L.G. Hector Jr, J.R. Fekete, W. Tong, Y. Xuan, Spatio-temporal characteristics of the Portevin-Le Châtelier effect in austenitic steel with twinning induced plasticity, International Journal of Plasticity, 25 (2009) 2298-2330.

[14] N. Ranc, D. Wagner, Some aspects of Portevin-Le Chatelier plastic instabilities investigated by infrared pyrometry, Materials Science and Engineering: A, 394 (2005) 87-95.

[15] X. Wang, L. Wang, M. Huang, Kinematic and thermal characteristics of Lüders and Portevin-Le Châtelier bands in a medium Mn transformation-induced plasticity steel, Acta Materialia, 124 (2017) 17-29.

[16] J. Florando, M. LeBlanc, D. Lassila, Multiple slip in copper single crystals deformed in compression under uniaxial stress, Scripta Materialia, 57 (2007) 537-540.

[17] E. Charkaluk, R. Seghir, L. Bodelot, J.-F. Witz, P. Dufrénoy, Microplasticity in Polycrystals: A Thermomechanical Experimental Perspective, Experimental Mechanics, 55 (2015) 741-752. 
[18] X.G. Wang, J.F. Witz, A. El Bartali, A. Oudriss, R. Seghir, P. Dufrénoy, X. Feaugas, E. Charkaluk, A Dedicated DIC Methodology for Characterizing Plastic Deformation in Single Crystals, Experimental Mechanics, 56 (2016) 1155-1167.

[19] A. Morabito, A. Chrysochoos, V. Dattoma, U. Galietti, Analysis of heat sources accompanying the fatigue of 2024 T3 aluminium alloys, International Journal of Fatigue, 29 (2007) 977-984.

[20] V. Crupi, G. Epasto, E. Guglielmino, A. Squillace, Influence of Microstructure [Alpha+ Beta and Beta] on Very High Cycle Fatigue Behavior of Ti-6Al-4V Alloy, International Journal of Fatigue, 95 (2017) 64-75.

[21] X.G. Wang, E.S. Feng, C. Jiang, A microplasticity evaluation method in very high cycle fatigue, International Journal of Fatigue, 94 (2017) 6-15.

[22] N. Ranc, D. Wagner, P. Paris, Study of thermal effects associated with crack propagation during very high cycle fatigue tests, Acta Materialia, 56 (2008) 4012-4021.

[23] R. Ghorbani, F. Matta, M.A. Sutton, Full-field deformation measurement and crack mapping on confined masonry walls using digital image correlation, Experimental Mechanics, 55 (2015) 227-243.

[24] S. Alkan, P. Chowdhury, H. Sehitoglu, R.G. Rateick, H.J. Maier, Role of nanotwins on fatigue crack growth resistance-Experiments and theory, International Journal of Fatigue, 84 (2016) 28-39.

[25] D. Favier, H. Louche, P. Schlosser, L. Orgéas, P. Vacher, L. Debove, Homogeneous and heterogeneous deformation mechanisms in an austenitic polycrystalline Ti-50.8at.\% Ni thin tube under tension. Investigation via temperature and strain fields measurements, Acta Materialia, 55 (2007) 5310-5322.

[26] L. Bodelot, E. Charkaluk, L. Sabatier, P. Dufrénoy, Experimental study of heterogeneities in strain and temperature fields at the microstructural level of polycrystalline metals through fully-coupled full-field measurements by digital image correlation and infrared thermography, Mechanics of Materials, 43 (2011) 654-670.

[27] X.G. Wang, J.F. Witz, A. El Bartali, C. Jiang, Infrared thermography coupled with digital image correlation in studying plastic deformation on the mesoscale level, Optics and Lasers in Engineering, 86 (2016) 264-274.

[28] A. Chrysochoos, V. Huon, F. Jourdan, J.M. Muracciole, R. Peyroux, B. Wattrisse, Use of Full-Field Digital Image Correlation and Infrared Thermography Measurements for the Thermomechanical Analysis of Material Behaviour, Strain, 46 (2010) 117-130.

[29] E. Toussaint, X. Balandraud, J.-B. Le Cam, M. Grediac, Combining displacement, strain, temperature and heat source fields to investigate the thermomechanical response of an elastomeric specimen subjected to large deformations, Polymer Testing, 31 (2012) 916-925.

[30] J.S. Martinez, X. Balandraud, E. Toussaint, J.-B. Le Cam, D. Berghezan, Thermomechanical analysis of the crack tip zone in stretched crystallizable natural rubber by using infrared thermography and digital image correlation, Polymer, 55 (2014) 6345-6353.

[31] J.S. Martinez, E. Toussaint, X. Balandraud, J.-B. Le Cam, D. Berghezan, Heat and strain measurements at the crack tip of filled rubber under cyclic loadings using full-field techniques, Mechanics of Materials, 81 (2015) 62-71.

[32] L. Bodelot, L. Sabatier, E. Charkaluk, P. Dufrénoy, Experimental setup for fully coupled kinematic and thermal measurements at the microstructure scale of an AISI 316L steel, Materials Science and Engineering: A, 501 (2009) 52-60. 
[33] S. Dumoulin, H. Louche, O. Hopperstad, T. Børvik, Heat sources, energy storage and dissipation in high-strength steels: experiments and modelling, European Journal of Mechanics-A/Solids, 29 (2010) 461-474.

[34] R. Seghir, L. Bodelot, E. Charkaluk, P. Dufrénoy, Numerical and experimental estimation of thermomechanical fields heterogeneity at the grain scale of $316 \mathrm{~L}$ stainless steel, Computational Materials Science, 53 (2012) 464-473.

[35] W. Wang, R. Fruehmann, J. Dulieu-Barton, Application of digital image correlation to address complex motions in thermoelastic stress analysis, Strain, 51 (2015) 405-418.

[36] N. Cholewa, P. Summers, S. Feih, A. Mouritz, B. Lattimer, S. Case, A technique for coupled thermomechanical response measurement using infrared thermography and digital image correlation (tdic), Experimental Mechanics, 56 (2016) 145-164.

[37] M. Silva, G. Ravichandran, Combined thermoelastic stress analysis and digital image correlation with a single infrared camera, The Journal of Strain Analysis for Engineering Design, (2011) 0309324711418286.

[38] A. Maynadier, M. Poncelet, K. Lavernhe-Taillard, S. Roux, One-shot measurement of thermal and kinematic fields: Infrared image correlation (iric), Experimental Mechanics, 52 (2012) 241-255.

[39] R. Montanini, F. Freni, Non-contact measurement of linear thermal expansion coefficients of solid materials by infrared image correlation, Measurement Science and Technology, 25 (2013) 015013.

[40] J. Westerweel, Fundamentals of digital particle image velocimetry, Measurement science and technology, 8 (1997) 1379.

[41] S. Klein, M. Staring, K. Murphy, M.A. Viergever, J.P. Pluim, Elastix: a toolbox for intensity-based medical image registration, IEEE Transactions on Medical Imaging, 29 (2010) 196-205.

[42] P. Cheng, M.A. Sutton, H.W. Schreier, S.R. McNeill, Full-field speckle pattern image correlation with B-spline deformation function, Experimental Mechanics, 42 (2002) 344-352.

[43] D. Rueckert, L.I. Sonoda, C. Hayes, D.L. Hill, M.O. Leach, D.J. Hawkes, Nonrigid registration using free-form deformations: application to breast MR images, IEEE Transactions on Medical Imaging, 18 (1999) 712-721.

[44] G. Bianchi, R. Sorrentino, Electronic filter simulation \& design, McGraw-Hill New York, 2007.

[45] T.M. Cover, J.A. Thomas, Elements of information theory, John Wiley \& Sons2012.

[46] A. Collignon, D. Vandermeulen, P. Suetens, G. Marchal, 3D multi-modality medical image registration using feature space clustering, Computer Vision, Virtual Reality and Robotics in Medicine, Springer, 1995, pp. 195-204.

[47] P. Viola, W.M. Wells III, Alignment by maximization of mutual information, International Journal of Computer Vision, 24 (1997) 137-154.

[48] F. Maes, A. Collignon, D. Vandermeulen, G. Marchal, P. Suetens, Multimodality image registration by maximization of mutual information, IEEE Transactions on Medical Imaging, 16 (1997) $187-198$. 